\title{
Verbalternationen und Verbspaltung im Neugriechischen
}

\author{
Chariton Charitonidis (Köln)
}

\begin{abstract}
This paper deals with a group of five alternations for the Modern Greek verb in the syntactic frame $\mathrm{NP}_{\mathrm{i}}$ _ $\mathrm{NP}$ for the active form and $\mathrm{NP}_{\text {_ }}$ for the corresponding active or passive form. The alternations causative/auto, causative/reflexive, causative/reciprocal, causative/control, and passive participle are presented. It is shown how the interpretation of some of these alternations is influenced by the context. The second part deals with the splitting of the verbs kapnizo and potizo into homophonous lexical units and explains why the alternations component must be favoured in comparison to the conceptual structures and semantic fields component. The proposed model incorporates a version of Ray Jackendoff's conceptual structures.
\end{abstract}

\section{$1 \quad$ Einleitung}

Damit Alternationen, i. e. reguläre semantische Relationen zwischen lexikalischen Einheiten, beschrieben werden können, muß die ganze Grammatik einer Sprache beachtet werden, d. h. ihre Morphologie, Syntax und möglicherweise auch ihre Phonologie (vgl. Behrens 1994: $161)$.

Ein griechisches Verb wie fanatízo ${ }^{1}$ 'fanatisieren' ist in anderen Mustern als das entsprechende englische Verb fanaticize eingebettet, nämlich Kausativ-Aktiv/Auto-Passiv (siehe fanatízo/fanatízome) bzw. Kausativ-Aktiv/Intransitiv²-Aktiv (siehe fanaticize/fanaticize), weil die Grammatik in beiden Sprachen anders organisiert ist.

Auf der Basis dieser Feststellungen werde ich im ersten Abschnitt eine Gruppe von Alternationen vorstellen, die im Neugriechischen die Lesarten von Verben adäquat abgrenzen, wie auch eine Gruppe von opaken Verbalternationen, die die relativierende Wirkung des Kontextes nahelegen.

Im zweiten Abschnitt werde ich am Beispiel der Verben kapnízo und potízo den regulären Charakter der Spaltung der Verblesarten zeigen, besonders in Bezug auf die im ersten

\footnotetext{
${ }^{1}$ Die Standard-Zitierform für griechische Verben ist die 1. Person Singular Aktiv (bzw. Passiv, wenn das Verb keine aktiven Formen hat).

2 Der Terminus Intransitiv wird hier als ein allgemeiner Terminus für die Alternationsvariante verwendet, die der Bedeutung 'als Fanatiker handeln' entspricht.
} 
Abschnitt vorgestellten Alternationen. Konzeptuelle Strukturen und semantische Felder (Situationsfelder) werden zur Vervollständigung der Semantik beider Verben herangezogen.

Die Analyse beschränkt sich mit drei Ausnahmen (siehe Abschnitt 2.1.4) auf die Verben auf izo im syntaktischen Rahmen $\mathrm{NP}_{\mathrm{i}}$ _- NP bei der aktiven Form und NP _- bei der entsprechenden aktiven oder passiven Form. ${ }^{3}$

\section{$2 \quad$ Verbalternationen}

\subsubsection{Kausativ-Aktiv / Auto-Aktiv (Alternation 1 a/b) Kausativ-Aktiv / Auto-Passiv (Alternation 2a/b)}

In dieser Alternation initiiert ein Agens ein Ereignis (kausative Variante) und dieses Ereignis kann unabhängig von diesem Agens konzeptualisiert werden (Auto-Variante). (1) ist ein Beispiel für die Alternation $1 a / b$ und (2) ist ein Beispiel für die Alternation $2 a / b$.

(1) a. I zésti ksínise to gála.

'Das warme Wetter hat die Milch sauer werden lassen'.

b. To gála ksínise.

'Die Milch ist sauer geworden (ist versauert)'

(2) a. I adipolítefsi midhenízi tis prospáthies tis kivérnisis.

'Die Opposition macht die Bemühungen der Regierung zunichte'.

b. I prospáthies tis kivérnisis midhenízode.

'Die Bemühungen der Regierung werden zunichte gemacht'.

Das Agens in Beispiel (1a), d. h. das warme Wetter, ist in Beispiel (1b) nicht präsent; die Milch kann ohne die Intervention eines kontrollierenden Agens (siehe Abschnitt 1.1.4) sauer werden, z. B. wenn die Säuerung über eine längere Zeit hinweg stattfindet. Außerdem kann ein Agens wie das warme Wetter oder die Luft einen Säuerungsprozeß initiiert haben, aber die Anwesenheit dieses Agens im Verlauf oder am Ende dieses Prozesses ist optional, d. h. das betreffende Ereignis wird als autonom konzeptualisiert.

Ähnlicherweise ist das Agens in Beispiel (2b), d.h. die Opposition, nicht präsent; die Bemühungen der Regierung können ohne die Intervention eines kontrollierenden Agens zunichte gemacht werden, z. B. wenn diese Bemühungen in einer Zeit finanzieller Krise stattfinden. Oder ein Agens kann lediglich einen Vernichtungsprozeß initiiert haben, wie im Fall einer Schmähkampagne; die Anwesenheit dieses Agens im Verlauf oder am Ende dieses Prozesses ist optional, i. e. das betreffende Ereignis wird als autonom konzeptualisiert.

\footnotetext{
3 Die Korpus- und Internetbelege wie auch die Internet-Links, die in diesem Aufsatz auftreten, haben keinen ideologischen oder politischen Bezug. Sie wurden lediglich zur Veranschaulichung der linguistischen Phänomene eingesetzt, die in diesem Aufsatz besprochen werden.
} 
Auto-Verben enthalten meistens ein implizites temporales Ende, z. B. denotiert die passive Auto-Variante des Verbs midhenízo in Beispiel (2b) ein Ereignis mit einem impliziten temporalen Ende: eine Regierungsbemühung kann nicht endlos vernichtet werden. Und wenn diese Regierungsbemühung einmal vernichtet ist, erwartet niemand weitere Vernichtungsstufen.

Dieselbe Argumentation gilt auch für Psych-Verben, d. h. Verben, die einen psychischen Zustand bezeichnen, vgl. Beispiel (3), das die Alternation $2 a / b$ enthält.

(3) a. Orjízun ton kósmo me ta psématá tus.

'Sie verärgern das Volk mit ihren Lügen'.

b. O kósmos orjízete me ta psématá tus.

'Das Volk wird durch ihre Lügen verärgert'.

Die Anwesenheit eines Agens im Verlauf oder am Ende des 'Verärgerungs-Ereignisses ist optional, vgl. die Auto-Variante in Beispiel (3b). Man kann sich ärgern, wenn man sich auch nur an die Lügen der anderen erinnert. Außerdem ist das Ereignis in Beispiel (3) zeitlich begrenzt, da es mit Kulmination und Ausklang eines psychischen Zustands verbunden ist.

In der Auto-Alternation wird der Prozeß des Zustandswechsels hauptsächlich auf der Basis der physischen, psychischen (wie im Fall der Psych-Verben) usw. Konstitution des Referenzobjektes definiert, z. B. ist eine Regierungsbemühung leichter als ein Gerichtsurteil $\mathrm{zu}$ vernichten (vgl. Beispiel (2)). Ein ruhiger Mensch ärgert sich nicht wie ein reizbarer Mensch oder ein Junge, wegen der verschiedenen Entwicklung oder des verschiedenen Zustands ihrer Nervensysteme (vgl. Beispiel (3)).

Der Haupttest für die Vorlage der Auto-Alternation (nicht immer anwendbar!) ist die Verwendung der Phrase (apó) mónos tu 'von alleine' (Mask.), (apó) móni tis 'von alleine' (Fem.), (apó) móno tu 'von alleine' (Neutr.), usw. bei der Auto-Variante (vgl. die Verwendung desselben Tests in Smith (1978: 104)).

In den meisten Fällen ist der Austausch der Phrasen apó+NP und mé+NP ein syntaktischer Beweis für das Vorliegen der Alternationen 1 und 2, vgl. mávrise apó ton kapnó und mávrise me ton kapnó '(es) wurde schwarz vom Rauch' (siehe Alternationsvariante 1 b), orjízete me ta psématá tus und orjízete apó ta psématá tus '(er) wird durch ihre Lügen verärgert' (vgl. Beispiel (3b)), die semantisch/pragmatisch äquivalent sind.

Zusätzlicher Beweis für das Vorliegen der Auto-Variante ist die Manifestation der true inchoative-Lesart ${ }^{4}$ im Präsens, z. B. kann die Auto-Variante des Verbs mavrízo 'schwarz werden' im Präsens 'anfangen, schwarz zu werden' bedeuten.

\footnotetext{
${ }^{4}$ Ich verwende den Terminus true inchoative, weil ich dieses Inchoativ, das den Beginn eines Ablaufs denotiert, von verschiedenen syntaktischen oder semantischen Definitionen abgrenzen will, vgl. die syntaktische causative/inchoative-Alternation in Levin (1993: 27ff) oder die semantische Definition eines inchoativeEreignisses in Jackendoff (1990: 91ff).
} 


\subsubsection{Kausativ-Aktiv / Reflexiv-Aktiv (Alternation 3a/b) Kausativ-Aktiv / Reflexiv-Passiv (Alternation 4a/b)}

In dieser Alternation initiiert ein Agens ein Ereignis (kausative Variante), in dem das Ziel seiner Kontrollhandlung (siehe Abschnitt 2.1.4) er selbst ist oder ein Teil von ihm (reflexive Variante). Das Satzpaar in Beispiel (4) veranschaulicht die Alternation 4a/b.

(4) a. Xtenízi ta maliá tis.

'Sie kämmt ihr Haar'.

b. Xtenizete.

'Sie kämmt sich'.

Alternation 3 kann nicht leicht für gültig erklärt werden, sie ist aber theoretisch möglich. Eine aktive Reflexivvariante (i. e. Alternationsvariante $3 b$ ) dürfte im folgenden Satz belegt werden:

(5) I paréa skórpise.

'Die Gesellschaft zerstreute sich'. (i. e. 'ging auseinander').

Das Problem bei Sätzen wie diese in Beispiel (5) ist, daß es keine entsprechende Kausativ-Variante gibt (i. e. Alternationsvariante $3 a$ ), die sowohl eine Agens-NP als auch eine Empfänger-NP mit derselben Referenz beinhalten könnte (siehe Beispiel (6).

(6) *I paréa skórpise ton eaftó tis.

'Die Gesellschaft zerstreute sich'.

Nur in einer konzeptuellen Struktur im Identifikationsfeld ('identificational field', siehe Jackendoff 1983: 194-198) kann eine reflexive Konfiguration für gültig erklärt werden, vgl. die LCS 5 in Beispiel (7), die sowohl für die reflexive als auch für die kausative Variante gilt.

(7) CAUSE([ Thing $_{\text {PARÉA }],\left[G_{\text {Ident }}\left([\text { Thing } P A R E ́ A],\left[\text { Path }_{\text {Ident }}[\text { Property }\right.\right.\right.}$ SKÓRPIOS $\left.\left.\left.\left.]\right]\right)\right]\right)$,

wo [SKÓRPIOS] für den Inhalt des rückgebildeten Adjektivs skórpios 'zerstreut' steht. CAUSE, GO und TO sind konzeptuelle Funktionen und Thing, Path und Property konzeptuelle Konstituenten. Für Einzelheiten über die Konzeptuelle Struktur als Grammatikebene siehe Jackendoff $(1983,1990,1992)$.

\subsubsection{Kausativ-Aktiv / Reziprok-Aktiv (Alternation 5a/b) Kausativ-Aktiv / Reziprok-Passiv (Alternation 6a/b)}

In dieser Alternation initiiert ein Agens ein Ereignis (kausative Variante), in dem das Ziel der Handlungen der Partizipanten aufeinanderzielen (reziproke Variante). Das Satzpaar in Beispiel (8) veranschaulicht die Alternation $6 a / b$.

${ }^{5}$ LCS = Lexical Conceptual Structure (dt. Lexikalische Konzeptuelle Struktur). 
(8) a. O Jórgos adíkrise ti Méri (oder) I Méri adíkrise ton Jórgo.

'Jórgos traf Mary' (oder) 'Mary traf Jórgos'. (wörtlich 'sah/befand sich gegenüber')

b. O Jórgos ke i Méri adikrístikan.

'Jórgos und Mary trafen einander'. (wörtlich 'sahen/befanden sich gegenüber')

Unter den von mir untersuchten Ableitungen auf -izo weisen nur wenige diese Alternation auf. Die meisten von ihnen sind alte Derivationen (vgl. xeretizo) oder opake Wörter (vgl. vrízo - siehe auch Charitonidis 2005).

Ähnlich wie bei Alternation $3 \mathrm{im}$ vorangehenden Abschnitt, kann Alternation 5 nicht leicht für gültig erklärt werden; sie ist aber theoretisch möglich. Eine aktive Reziprok-Variante (i. e. Alternationsvariante 5b) dürfte in Beispiel (9) belegt sein, welches das formlose Verb agapízo 'sich versöhnen' beinhaltet, abgeleitet vom Verb agapó 'lieben' über das AoristParadigma.

(9) I adízili agápisan.

'Die Rivalen haben sich versöhnt'. (wörtlich 'haben sich geliebt')

Ähnlich wie im Fall der aktiven Reflexiv-Variante im vorangehenden Abschnitt kann eine reflexive Konfiguration nur in einer konzeptuellen Struktur in einem erweiterten semantischen Feld für gültig erklärt werden, vgl. die LCS in Beispiel (10).

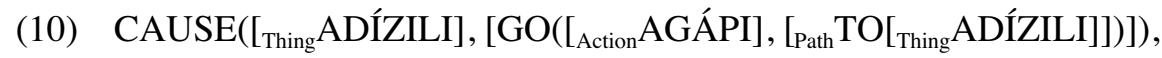

wo [AGÁPI] für den Inhalt des verwandten 6 Basisnomens agápi 'Liebe' steht.

\subsubsection{Kausativ-Aktiv / Kontroll-Aktiv (Alternation 7a/b) Kausativ-Aktiv / Kontroll-Passiv (Alternation 8a/b)}

In dieser Alternation initiiert ein Agens ein Ereignis (kausative Variante) und es hat die Kontrolle darüber, besonders indem es das Ende dieses Ereignisses bestimmt (KontrollVariante). (11) ist ein Beispiel für die Alternation $8 a / b$.

(11) a. O májiras alatízi to fajitó.

'Der Koch salzt das Essen'.

b. To fajitó alatízete apó ton májira.

'Das Essen wird vom Koch gesalzen'.

\footnotetext{
${ }^{6}$ Eine Derivationsbasis wie agápi wird als verwandt bezeichnet, wenn sie mit einer nicht direkten Ableitung wie agapízo, das eine Bildung aus dem Aorist-Paradigma von agapó 'lieben' ist, in Verbindung gebracht wird.
} 
In Beispiel (11) sind das Agens májiras 'Koch' und die bewegende Entität bzw. Thema ('theme') aláti 'Salz', repräsentiert durch die Basis des Derivates alatízo, unentbehrliche Entitäten in der vorliegenden Handlung. Das Agens hat als eine willentliche Entität Kontrolle über das ganze Ereignis, indem es Ablauf und Ende dieses Ereignisses bestimmt.

(12) ist ein Beispiel für die viel seltener auftretende Alternation 7a/b.

(12) a. I néa mamá megalóni to agoráki.

'Die junge Mutter erzieht den kleinen Jungen'. (wörtlich 'macht groß')

b. To agoráki megalóni apó ti néa mamá tu.

'Der kleine Junge wird von seiner jungen Mutter erzogen'.7 (wörtlich 'wird groß gemacht')

Ähnlich wie in Beispiel (11) initiiert in Beispiel (12) das Agens i néa mamá 'die junge Mutter' ein Erziehungsereignis (kausative Variante 7a) und es hat die Kontrolle darüber, besonders indem es das Ende dieses Ereignisses bestimmt (Kontroll-Variante $7 b){ }^{8}$

Wir sehen also, daß das Agens im Gegensatz zu den Alternationen $1 a / b$ und $2 a / b$ immer präsent im zweiten Alternationsglied ist. Außerdem ist die ganze Alternation in Bezug auf die explizite oder implizite Anwesenheit der Hauptargumente in der konzeptuellen Struktur symmetrisch.

In diesem Aufsatz wird nicht der Versuch unternommen, eine syntaktische Darstellung der Alternationen in Bezug auf die Oberflächenargumente zu bieten, sondern dafür eine semantisch-pragmatische auf der Basis von Handlungsschemata. Zum Beispiel ist die apó-Phrase in der $b$-Variante abwesend in vielen Konstruktionen mit Kontrollverben (siehe HNC-Korpus und Internet). Im Falle von alatizo and anderen Verben mit einem Basisnomen als Thema ist das auf das Foregrounding der Thema-Bewegung zurückzuführen, so daß das Agens in der Syntax nicht erscheint. Das bedeutet aber nicht, daß das Agens während des betreffenden Ereignisses in der konzeptuellen Struktur fehlt (vgl. Jackendoff 1990: 53).

Darüber hinaus können Kontrollverben den true inchoative-Test in der $b$-Variante nicht bestehen (siehe Abschnitt 2.1.1). Ein Verb wie vurtsízo 'bürsten' kann nicht 'anfangen gebürstet zu werden' im Präsens Passiv bedeuten.

7 Die Lesart 'großgezogen/erzogen werden' des Verbs megalóno (siehe Alternationsvariante $7 b$ ) muß im Einklang mit dem hier vorgestellten Alternationsmodell von der verwandten Lesart 'heranwachsen' derselben Verbform (siehe Alternationsvariante $1 a$ ) abgegrenzt werden.

8 Zwei weitere Belege für die Alternationsvariante $7 b$ sind in Beispiel (i) und (ii) aufgeführt.

(i) O xaraktíras tu íroá tu isoropí apó ton metriméno Liam Neeson.

'Der Charakter seines Helden wird vom gemessenen Liam Neeson in Gleichgewicht gebracht' (http://www.e-shop.gr/show_dvd.phtml?id=DVD.01800: Stand: 9.11.2005).

(ii) I Patisíon klíni apó mathités ke ergátes pu katevénun se sibarástasi.

'Die Patission-Straße wird von Studenten und Arbeitern abgesperrt (wörtlich 'schließt'), die zum Beistand zusammenlaufen' (http://www.dea.org.gr/efhmerida/86/keimeno4.htm: Stand: 9.11.2005). 


\subsubsection{Passives Partizip (Alternation 9)}

Diese Alternation hat nur ein Glied. Sie denotiert einen etablierten Endzustand, d. h. sie bezieht sich auf einen erreichten Zustand mit einer temporalen Erweiterung nach Abschluß des betreffenden Ereignisses. Ein Beispiel für diese Alternation ist unter (13) aufgeführt.

\section{(13) To psári íne tiganisméno. \\ 'Der Fisch ist (in der Pfanne) gebraten'.}

In den meisten Fällen weisen Verben auf -izo, die kein passives Partizip haben, die Alternationen 1-8 nicht auf (siehe die Verben fterujízo, travlízo u.a. in Charitonidis 2005). Das passive Partizip ist folglich ein Indiz auf das Vorliegen dieser Alternationen.

\subsection{Multimapping}

Die mehrfache Abbildung von Semantikkomponenten auf Morphologieeinheiten, hier Multimapping genannt, kommt im Bereich der Verbderivation im Neugriechischen grundsätzlich dadurch zur Geltung, daß für den Ausdruck derselben Verbbedeutung parallele Suffixe mit -izo konkurrieren (siehe Alternationsklassen in Charitonidis 2005: 87-130). In diesem Abschnitt werde ich mich mit dem Multimapping der Verbsemantik auf die aktive und/oder passive Morphologie auseinandersetzen.

Diathesenveränderungen des neugriechischen Verbs entsprechen nicht immer einer unterschiedlichen Semantik, insofern sie manchmal auf dasselbe Ereignis verweisen, siehe folgende Sätze:

(14) Ta skupídhia skórpisan. (Auto-Variante $1 b$ in Aktiv)

'Der Müll zerstreute sich'.

(15) Ta skupídhia skorpístikan. (Auto-Variante $2 b$ in Passiv)

'Der Müll zerstreute sich'.

(16) Ta skupídhia skorpístikan apó ti gáta. (Kontrollvariante $8 b$ in Passiv)

'Der Müll wurde von der Katze verstreut'.

Wie wir sehen, verweisen die aktive Form skórpisan in Beispiel (14) und die passive Form skorpístikan in Beispiel (15) auf dasselbe Ereignis. Andererseits kann die passive Form skorpístikan ein Auto- oder Kontrollereignis denotieren, vgl. Beispiel (15) bzw. (16).

Die Kontrollkategorie wird in der Regel durch Passiv ausgedrückt. Die Tatsache, daß Sätze wie (17) unten nicht von allen Informanten als ungrammatisch bewertet werden, ist noch ein Anzeichen dafür, daß es Aktiv oder Passiv nicht immer gelingt, eindeutige semantische Funktionen zu übernehmen (siehe Abschnitt 1.1.4 und Fußnoten 7, 8 für Internet-Belege). 
(17) ?Ta skupídhia skórpisan apó ti gáta. (Kontrollalternation $7 b$ in Aktiv)

'Der Müll wurde von der Katze verstreut'.

In den Alternationsklassen (vorgestellt in Charitonidis 2005: 87-130) habe ich versucht, alle Fälle von Diathesen-Multimapping aufzunehmen. Sie weisen unter anderem darauf hin, das sich Aktiv und Passiv im Bereich des neugriechischen Verbsystems oft indifferent überlappen.

\subsection{Opake Alternationen}

In Abschnitt 1.1 habe ich auf der Basis von rekurrenten Entsprechungen zwischen Semantik und Morphosyntax eine Gruppe von Verbalternationen definiert. Die semantische Basis ist jedoch nicht immer deutlich. Der Kontext entscheidet in vielen Fällen die Interpretation bestimmter Verben, so daß diese Verben in die Domäne der opaken Alternationen fallen. Im folgenden werden drei von diesen opaken Alternationen besprochen.

\subsubsection{Auto-/Reflexivalternation}

In diesem Abschnitt wird der opake Charakter der Alternationsvariante $3 b$ diskutiert, wie er sich in Beispiel (18) offenbart.

(18) I paréa skórpise.

'Die Gesellschaft hat sich zerstreut'.

In einer Erstinterpretation wirkt ein willentliches Agens ('volitional agent') auf sich selbst. In diesem Fall legt das Verb die Bewegungsinterpretation nahe, die mit der Tatsache verbunden ist, daß das Agens i paréa sich an einen anderen Ort begibt.

In einer zweiten Interpretation, wobei ein Ereignis von einem Beobachter berichtet wird, zum Beispiel von einem, dessen Freunde nicht mehr in seinem Blickfeld sind, bezeichnet (18) ein Auto-Ereignis, d.h. das Ereignis des Zerstreuens/Verschwindens, und die Bewegungsinterpretation ist sekundär bzw. wird nicht belegt. In diesem Fall kann der Kontext eine von beiden Interpretationen in den Vordergrund stellen.

\subsubsection{Auto-/Kontrollalternation}

Es gibt eine Verbgruppe, die nicht ein völlig autonomes Ereignis denotiert. Bei diesen Verben ist die Präsenz eines Agens, das das Ereignis unter Kontrolle hat, möglich. (19) veranschaulicht diesen Fall. 
(19) Ja tis metrísis aftés ta dhígmata thrimatístikan se komátia mikrótera apó $1 \mathrm{~cm}^{3}$ étsi óste na eksasfalísume óti ólo to radhónio to opío isérxete stus pórus na eklíete apó to dhígma. (HNC 1078284)

'Zu diesen Messungen wurden die Proben in Stücke kleiner als $1 \mathrm{~cm}^{3}$ fragmentiert, so daß gesichert werden kann, daß das ganze Radon, das in die Poren eindringt, aus der Probe ausgesondert wird'.

Das passive Verb thrimatístikan in Beispiel (19) wird eher als ein Auto-Verb qualifiziert. Es verweist auf ein Ereignis mit einem impliziten temporalen Ende, in dem ein Agens einen Prozeß in Gang setzt, der danach eine autonome Dynamik entwickelt. Es ist die explizite Intervention eines Agens in den mittleren Stufen dieses Ablaufs, die auf die Existenz einer Kontrollkomponente verweist, vgl. die NP komátia mikrótera apó $1 \mathrm{~cm}^{3}$ 'Stücke kleiner als 1 $\mathrm{cm}^{31}$ und die VP na eksasfalísume 'kann gesichert werden', die eine Kontrollinterpretation des Verbs nahelegen.

Andererseits wird in Beispiel (20) die Auto-Komponente in den Vordergrund gestellt.

(20) A ná tiganístike kiólas. Étimo to piáto mas!9

'Hey, es ist schon gar (wörtlich 'ist gebraten'). Das Gericht ist fertig!'

Obwohl tiganizo ein Kontrollverb ist (vgl. die Derivationsbasis tigáni 'Bratpfanne', die ein Instrument denotiert), werden vom Kontext die konstitutiven Eigenschaften eines impliziten Themas wie psári 'Fisch' in den Vordergrund gestellt, so daß das Ereignis, das im ersten Satz von (20) enthalten ist, weitgehend als autonom erfaßt wird.

\subsubsection{Komplementär in den Vordergrund gestellte Komponenten}

Es gibt eine Gruppe von Verben, die eine Auto- und eine Kontrollkomponente enthalten, die dem Kontext gemäß komplementär in den Vordergrund gestellt werden. In folgenden Sätzen kann das Verb stigmatízo bedeuten:

a. 'beflecken'

(21) Ki aftó fánike perítrana stis dhimotikés ke nomarxiakés eklojés i opíes stigmatístikan apó éna órjio paraskiniakón dhiavuléfseon... ${ }^{10}$

'Und das wurde ganz ohne Zweifel klar bei den Kommunal- und Landtagswahlen, die durch eine Orgie von Absprachen hinter den Kulissen befleckt wurden...'

\footnotetext{
${ }^{9}$ Siehe http://www.pcmaster.gr/sintages/default.asp?Show=r2 (Stand: 9.11.2005).

${ }^{10}$ Siehe http://www.hri.org/E/1998/98-11-19.dir/stiles/analisi.htm (Stand: 9.11.2005).
} 
b. 'stigmatisieren', 'brandmarken'

(22) i prósfijes pu kataskinónun ékso apó to xorió, apefthínode mátea stus xorikús ja voíthia, ke teliká ótan aftí dhen tus paréxete epitíthede, stigmatízode apó tus xorikús ke tus Túrkus san Bolsevíki. ${ }^{11}$

'...die Flüchtlinge, die außerhalb des Dorfes kampieren, bitten die Dorfbewohner vergebens um Hilfe, und wenn sie angreifen, weil sie (i. e. die Hilfe) ihnen nicht gewährt wird, werden sie von den Dorfbewohnern und den Türken als Bolschewiken gebrandmarkt'.

Der Einsatz des Verbs stigmatízo in Beispiel (22) als Verb des Sprechens stellt die Kontrollinterpretation in den Vordergrund. Das passiert, weil, wenn man spricht, man Kontrolle über die ganze Handlung des Sprechens hat. Das ist nicht der Fall bei (21), in dem keine Agenskontrolle zur Geltung kommt.

Ähnliche Verben sind sixtirízo und tonízo.

\subsubsection{Reflexiv-/Kontrollalternation}

Beim Vorkommen zweier Agenzien in einem Ereignis ist nicht immer klar, welches von beiden die vom Verb denotierte Handlung initiiert, während es möglich ist, daß sich beide Agenzien an der Handlung gleichzeitig beteiligen. Siehe dazu folgendes Beispiel:

(23) Sto metaksí i nífi stolízete ke etimázete sto spíti tis. Kopéles... katá ton stolismó léne dhiáfores madinádhes... ${ }^{12}$

'Inzwischen wird die Braut gekleidet (bzw. kleidet sich die Braut) und sie wird vorbereitet (bzw. bereitet sich) bei ihr zu Hause (vor). Mädchen... singen beim Ankleiden verschiedene madinádhes ${ }^{13}$...'

Im ersten Satz von (23) mögen zwei Agentien am Ereignis teilnehmen: das höhere Agens (der Familienkreis), das die Braut ankleidet, und das niedrigere Agens (die Braut), die sich kleidet. Nach der Standardinterpretation, initiiert das höhere oder das niedrigere Agens die erste Ereignisetappe und danach sind beide Agenzien gleichzeitig aktiv. Die Interpretation, wonach das niedrigere Agens die ganze Handlung initiiert und ausführt, ist marginal. Es liegt am Kontext wieder, ob die eine oder andere Interpretation in den Vordergrund (oder Hintergrund) gestellt wird.

Ähnliche Verben sind afionízo und oplízo. (reflexive Variante).

\footnotetext{
${ }^{11}$ Siehe http://www.historical-museum.gr/kazantzakis/gr/beaton.html (Stand: 9.11.2005).

12 Siehe http://www.wedsite.gr/tradition/tradition.asp?tradID=18 (Stand: 9.11.2005).

13 Madinádhes sind Volkscouplets, die auf der Insel Kreta gesungen werden.
} 


\section{Gespaltene Verben}

Eine direkte Folge der Festlegung einer Gruppe von Alternationen, an denen die neugriechischen Verben teilnehmen, ist die Spaltung einiger Verben in getrennte und autonome Lesarten, d.h. getrennte Verben. Die Untersuchung der Faktoren, die zu einer solchen Spaltung führen, ist sehr wichtig, weil sie erklärt, wie phonologisch identische Verben auf der Basis von Alternationen differenziert werden können, und weil sie Interpretationsmechanismen aufdeckt, die in allen Verben, transparenten und nicht transparenten, involviert sind.

In Beispiel (24) werden zwei LCS vorgestellt, die zum Profil der in Abschnitt 2.1 und 2.2 behandelten Verben beitragen sollen.

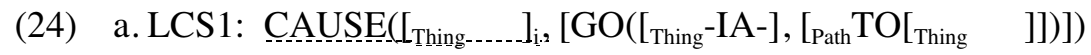

$$
\begin{aligned}
& \text { b. LCS4: CAUSES( }\left[_{\text {Thing........ }]_{i, 2}}\left[\mathrm{GO}\left(\left[_{\text {Thing }}-\mathrm{IA}-\right],\left[\mathrm{FROM}\left[_{\text {Thing }} \quad\right]\right]\right)\right]\right)
\end{aligned}
$$

wo 'IA' für das inkorporierte Argument steht. Die gebrochene Linie unter CAUSE weist darauf hin, daß das Agens in der Alternationsvariante $b$ fehlen kann. Ein Inventar konzeptueller Strukturen, die in der neugriechischen Verbderivation auftauchen, ist in Charitonidis (2005) zu finden.

\subsection{Gespaltene Verben mit transparenten Strukturen}

In Tabelle 1 werden die drei wichtigsten Lesarten des Verbs kapnízo in Bezug auf ihre syntaktische, semantische und morphologische Zusammensetzung präsentiert.

Tabelle 1

\begin{tabular}{|c|c|c|c|c|}
\hline Verben & Lesarten & Alternationsklassen & Semantische Felder & $\begin{array}{l}\text { Konzeptuelle } \\
\text { Strukturen }\end{array}$ \\
\hline kapnízol & $\begin{array}{l}\text { 'rauchen', 'Rauch } \\
\text { ausgeben' }\end{array}$ & $\begin{array}{l}1 * a / b_{-} * 9 \\
\text { (Keine } \\
\text { Alternationen) }\end{array}$ & $\begin{array}{l}\text { EMISSION/ } \\
\text { ENDOGENES } \\
\text { PRODUKT }\end{array}$ & $\begin{array}{l}\text { LCS4 } \\
\text { (siehe (24b)) }\end{array}$ \\
\hline kapnízo2 & 'räuchern' & $2 a / b \_8 a / b \_9$ & DECKUNG & $\begin{array}{l}\text { LCS1 } \\
(\text { siehe (24a)) }\end{array}$ \\
\hline kapnízo3 & $\begin{array}{l}\text { 'rauchen' (z. B. eine } \\
\text { Zigarette) }\end{array}$ & 8a/b_9 & $\begin{array}{l}\text { EMISSION/ } \\
\text { ENDOGENES } \\
\text { PRODUKT }\end{array}$ & $\begin{array}{l}\text { LCS4 } \\
\text { (siehe (24b)) }\end{array}$ \\
\hline
\end{tabular}

$1 * a / b_{-} * 9: *$ Kausativ-Aktiv/Auto-Aktiv_*Passives Partizip

2a/b_8a/b_9: Kausativ-Aktiv/Auto-Passiv_Kausativ-Aktiv/Kontroll-Passiv_Passives Partizip

8a/b_9: Kausativ-Aktiv/Kontroll-Passiv_Passives Partizip

Alle drei Verben in Tabelle 1 sind spezialisiert, insofern ihre Lesarten mit drei verschiedenen Szenen fest verbunden sind: 


\section{Szene 1}

Kapnízol denotiert die EMISSION von Rauch (siehe das inkorporierte Argument/Thema [KAPNÓS]) aus dem Inneren eines Referenzobjektes, z. B. eines Scheiterhaufens (siehe [FOTIÁ]). Die konzeptuelle Struktur für diese Lesart ist in Beispiel (25) angeführt.

$$
\text { GO([Thing KAPNÓS], [FROM[ } \left.\left[_{\text {Thing }} \text { FOTIÁ] }\right]\right)
$$

Dieses Ereignis wird unabhängig von einem Agens (siehe Auto-Variante $1 b$ mit aktiver Morphologie) und von einem etablierten Endzustand konzeptualisiert (ein passives Partizip wurde für diese Lesart nicht belegt). Aus diesem Grund sind Phrasen wie *kapnízi ti fotiá 'er raucht den Scheiterhaufen' (siehe Alternationsvariante 1a) bzw. *kapnisméni fotiá 'gerauchter Scheiterhaufen' (siehe Alternation 9) ungrammatisch.

\section{Szene 2}

Kapnízo2 denotiert die DECKUNG eines Referenzobjektes, z. B. einer Wand (siehe das Referenzobjekt [TÍXOS]) mit Rauch (siehe das inkorporierte Argument/Thema [KAPNÓS]). Die konzeptuelle Struktur für diese Lesart ist in Beispiel (26) aufgeführt.

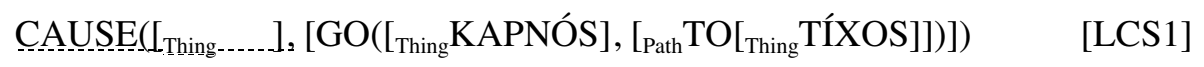

Wie die gebrochene Linie im Beispiel (26) zeigt, ist das erste Argument von CAUSE optional, d.h. das Ereignis kann unabhängig von einem Kontrollagens konzeptualisiert werden (siehe Auto-Variante $2 b$ mit passiver Morphologie). Zum Beispiel ist es nicht nötig, daß Phrasen wie o tíxos kapnístike 'die Wand war/ist verräuchert' ein Kontrollagens beim Ereignisablauf signalisieren, vgl. die verräucherten Fassaden von Gebäuden in viel befahrenen Straßen.

Andererseits muß ein Kontrollagens im Fall von Ereignissen wie das Anräuchern einer Wand mit einer Kerzenflamme präsent sein: der externe Initiator hat Kontrolle über die ganze Handlung und bestimmt auch ihr Ende (siehe Kontrollvariante $8 b$ mit passiver Morphologie).

Im Gegensatz zu kapnízo1 kann kapnízo2 einen etablierten Endzustand in seiner konzeptuellen Struktur enthalten, d. h. Phrasen wie kapnisménos tíxos 'verräucherte Wand' sind vollkommen grammatisch.

\section{Szene 3}

Kapnízo3, wie kapnízo1, denotiert die EMISSION von Rauch (siehe das inkorporierte Argument/Thema [KAPNÓS]), der als ein ENDOGENES PRODUKT aus dem Inneren eines Referenzobjektes, z. B. einer Zigarette (siehe [TSIGÁRO]) entsteht. Die konzeptuelle Struktur für diese Lesart ist in Beispiel (27) aufgeführt. 
Wie die LCS in Beispiel (27) zeigt, enthält kapnízo3 ein obligatorisches Agens im Gegensatz zu kapnízol und kapnízo2, d. h. der externe Initiator hat immer Kontrolle über die gesamte Handlung und bestimmt auch ihr Ende (siehe Kontrollvariante $8 b$ mit passiver Morphologie). Falls es keine Kontrolle gibt, z. B. wenn die Zigarette ohne die Anwesenheit eines Agens ausgeht, dann können wir nicht die Alternationen für kapnízo3 benutzen, um dieses Ereignis zu beschreiben. Kapnízo3 wie kapnízo2 enthält einen etablierten Endzustand in seiner konzeptuellen Struktur, d.h. Phrasen wie kapnisméno tsigáro 'gerauchte Zigarette' sind vollkommen grammatisch.

Wenn wir alle drei Verben näher betrachten, dann stellen wir fest, daß sie erst auf der Basis der Alternationsklassen, in denen sie auftreten, vollständig differenziert werden, und nicht auf der Basis der konzeptuellen Strukturen und/oder semantischen Felder, zu denen sie in Zusammenhang stehen.

Weitere gespaltene Verben mit transparenten Strukturen sind asfalízo, asprízo, axnízo, dhrosízo, ksinízo, midhenízo, orízo, plevrízo, prasinízo, progízo, sximatízo und termatízo (siehe Appendix A).

\subsection{Gespaltene Verben mit opaken Strukturen}

Tabelle 2 erläutert, wie die Zusammensetzung eines opaken Verbs wie potízo sein kann:

Tabelle 2

\begin{tabular}{|lllll|}
\hline Verben & Lesarten & Alternationsklassen & Semantische Felder & $\begin{array}{l}\text { Konzeptuelle } \\
\text { Strukturen }\end{array}$ \\
\hline potízo1 & 'bewässern' & $8 a / b \_9$ & WASSER & $\begin{array}{l}\text { opak } \\
\text { (s. Tabelle 3) }\end{array}$ \\
\hline potízo2 & 'tränken (ein Tier/ & $4 a / b \_8 a / b \_9$ & FLÜSSIGKEIT/ & $\begin{array}{l}\text { opak } \\
\text { (s. Tabelle 3) }\end{array}$ \\
\hline potízo3 & 'sickern' & $1 a / b \_2 a / b \_9$ & WASSER & $\begin{array}{l}\text { opak } \\
\text { (s. Tabelle 3) }\end{array}$ \\
\hline
\end{tabular}

8a/b_9: Kausativ-Aktiv/Kontroll-Passiv_Passives Partizip

4a/b_8a/b_9: Kausativ-Aktiv/Reflexiv-Passiv_Kausativ-Aktiv/Kontroll-Passiv_Passives Partizip la/b_2a/b_9: Kausativ-Aktiv/Auto-Aktiv_Kausativ-Aktiv/Auto-Passiv_Passives Partizip

Auf der Basis der Thema-Bewegung kann man die inkorporierten Argumente ableiten (vgl. die semantischen Felder in Tabelle 2). Nach dieser Identifizierung können die entsprechenden konzeptuellen Strukturen konstruiert werden, wie Tabelle 3 veranschaulicht (siehe das erste Argument der Funktion GO). 
Tabelle 3

\begin{tabular}{|c|c|c|c|c|}
\hline potízol & CAUSE( $\left(\left[_{\text {Thing }}\right.\right.$ & ],$\left[\mathrm{GO}\left(\left[_{\text {Thing }} \mathrm{WASSER}\right],{ }_{\text {Path }} \mathrm{TO}\left[_{\text {Thing }}\right.\right.\right.$ & & LCS1 \\
\hline potízo2 & CAUSE( $\left(\left[_{\text {Thing }}\right.\right.$ & ], [GO([Thing $F L U ̈ S S I G K E I T / W A S S E R],\left[{ }_{\text {Path }} \mathrm{TO}[\right.$ Thing & ])]) & LCS 14 \\
\hline potízo3 & CAUSE([Thing & $\begin{array}{r}\text { ], [GO([Thing } F L U ̈ S S I G K E I T / F E U C H T I G K E I T], \\
{\left[{ }_{\text {Path }} \mathrm{TO}\left[_{\text {Thing }}\right.\right.}\end{array}$ & ])]) & LCS $11^{15}$ \\
\hline
\end{tabular}

Die thematische Struktur aller drei Verben ist im Prinzip dieselbe. Das vollkommen inkorporierte Argument hat dieselbe strukturelle Position und drückt dieselbe ontologische Kategorie in derselben minimalen konzeptuellen Struktur ${ }^{16}$ aus, d. h. es ist ein Thing/Thema, das sich auf einer Pfadbahn zu einem anderen Thing/Referenzobjekt bewegt.

Die nächste Folge der Opazität dieser Strukturen ist, daß die relevanten semantischen Felder nicht zuverlässig bestimmt werden können, wie im Falle der transparenten Verben (siehe Charitonidis 2005: 77f). Obwohl eine erhebliche Ähnlichkeit zwischen den abgeleiteten inkorporierten Argumenten existiert, muß die genaue Definition dieser Argumente auf der Basis der Szenen vorgenommen werden, in denen die drei Verblesarten vorkommen. Diese Szenen müssen etwas wie 'Bewässern' für potízol, 'Transfer von Flüssigkeit/Wasser unter Menschen und/oder Tieren' für potízo2, und 'Penetration von Flüssigkeit/Feuchtigkeit in ein Material' für potízo3 sein. Den Ereignisrahmen bzw. die Ereignisstruktur dieser Szenen bieten die Alternationsklassen, i. e. die morphosyntaktische Zusammensetzung dieser drei Verben (siehe Tabelle 2). Es ist die sprachliche Ebene, auf der die drei Verblesarten explizit und adäquat differenziert werden.

\section{Schluß}

Die allgemeinen Erkenntnisse, die aus der Analyse in Abschnitt 1 und 2 gewonnen werden können, sind:

1. Im Neugriechischen ist die Art der Beteiligung eines Agens in Ereignissen wesentlich eine Frage der Verbmorphologie. Es gibt dazu eine Tendenz, daß eine aktive Verbform sich auf Ereignisse bezieht, für die üblicherweise die passive Morphologie vorbehalten ist (siehe Alternationsvarianten $3 b, 5 b$ und $7 b$ ). Ferner kann der Kontext die Alternationslesart einer Verbeinheit in regelmäßiger Weise beeinflussen (siehe Abschnitt 1).

2. Lexikalische Regeln für die Beschreibung des Verhältnisses zwischen einer Basis und einer Verbableitung sind ein Artefakt, verbunden mit dem Musteraufbau von konzeptuellen Strukturen innerhalb von Alternationsklassen (vgl. die konzeptuellen Strukturen von kapnízo

\footnotetext{
${ }^{14}$ Plus reflexives Binding (siehe Jackendoff 1990) für die reflexive Passivvariante $4 b$.

15 Plus Argumentfusion ('argument fusion') für die kausativen Varianten, d. h. das vollkommen inkorporierte Argument [FLÜSSIGKEIT/FEUCHTIGKEIT] funktioniert bei Sätzen wie i igrasía pótise ton tíxo 'die Feuchtigkeit ist in die Wand eingesickert' als eine Selektionsbeschränkung ('selectional restriction') für die Subjekt-NP i igrasía (zur Regel der Argumentfusion siehe Jackendoff 1990:53f).

16 Eine minimale konzeptuelle Struktur ist eine Struktur, die das thematische Hauptereignis ('main thematic Event') in den Vordergrund stellt (siehe Charitonidis 2005: 60-62 für Einzelheiten).
} 
in (25), (26) und (27)). Andererseits bekräftigen ähnliche Muster bei opaken Verben wie potizo diese Annahme (siehe Abschnitt 2.2) und sie zeigen, daß der entscheidende Unterscheidungsfaktor der Verblesarten im Neugriechischen die Zugehörigkeit zu einer Alternationsklasse ist. Mit anderen Worten markiert die Alternationsklasse den Inhalt der Derivationsbasis auf einer höheren Ebene, z. B. der Basisinhalt von kapnízol ist nicht lediglich [KAPNÓS], sondern [KAPNÓS] $+1 * a / b_{-} * 9$ und der Basisinhalt von kapnízo2 ist nicht lediglich wieder [KAPNÓS], sondern [KAPNÓS] + 2a/b_8a/b_9 usw., so daß die genauere Bezeichnung dieser Basis als Input für eine lexikalische Regel kooperativ erreicht werden kann.

\section{Literaturangaben}

Babiniótis, Jeórjios (1998): Leksikó tis Néas Elinikís Glósas ('Wörterbuch der neugriechischen Sprache'). Athen.

Behrens, Leila (1995): "Alternationen - ein Schlüssel für die Universalienforschung." Zeitschrift für Sprachwissenschaft 13/2: 149-200.

Charitonidis, Chariton (2005): Verb Derivation in Modern Greek: Alternation Classes, Conceptual Structures, Semantic Fields. Frankfurt.

Dhelijánis, Ioánis/Lipurlís, Dhimítris et al. (eds.) (1999): Leksikó tis Kinís Neoelinikís ('Wörterbuch des Standard-Neugriechischen'). Thesaloníki.

ILSP (2002): Hellenic National Corpus ${ }^{\mathrm{TM}}$ (HNC). Web Version 2.0 (http://hnc.ilsp.gr/).

Jackendoff, Ray (1983): Semantics and Cognition. Cambridge, MA.

Jackendoff, Ray (1990): Semantic Structures. Cambridge, MA.

Jackendoff, Ray (1992): "Parts and boundaries". In: Levin, Beth/Pinker, Steven (eds.) (1992): Lexical and Conceptual Semantics: 9-45.

Jazayery, Mohammed A./Polomé, Edgar C./Winter, Werner (eds.) (1978): Linguistic and Literary Studies in Honor of Archibald A. Hill [Vol. 2: Descriptive Linguistics]. The Hague.

Holton, David/Mackridge, Peter/Philippaki-Warburton, Irene (1997): Greek: a comprehensive grammar of the modern language. London.

Levin, Beth (1993): English Verb Classes and Alternations. Chicago.

Levin, Beth/Pinker, Steven (eds.) (1992): Lexical and Conceptual Semantics. Oxford.

Smith, Carlota S. (1978): "Jespersen's 'Move and Change' Class and Causative Verbs in English". In: Jazayery, Mohammed A./Polomé, Edgar C./Winter, Werner (eds.) (1978): Linguistic and Literary Studies in Honor of Archibald A. Hill [Vol. 2: Descriptive Linguistics]. The Hague: 101-109. 


\section{Appendix A: Verben auf -izo und Alternationsklassen}

\begin{tabular}{|c|c|c|}
\hline & Bedeutung & Alternationsklasse \\
\hline adikrízo & sehen/treffen & $6 a / b_{-} * 9$ \\
\hline afionízo & Opium verabreichen & $4 a / b \_8 a / ? b \_9$ \\
\hline alatízo & salzen & $8 a / b \_9$ \\
\hline asfalízol & einschließen, mit einem Schloß versehen & $8 a / b \_9$ \\
\hline asfalízo2 & versichern, assekurieren & $4 a / b \_8 a / b \_9$ \\
\hline asprízol & weiß machen & $1 a / b \_9$ \\
\hline asprízo2 & tünchen & $8 a / b \_9$ \\
\hline axnízol & ausdünsten, dampfen & $1 * a / b \_* 9$ \\
\hline axnízo2 & dünsten, dämpfen & $8 a / b \_9$ \\
\hline dhrosízol & kühlen & $1 a / b \_9$ \\
\hline dhrosízo2 & erfrischen & $2 a / b \_4 a / b \_9$ \\
\hline fanatízo & fanatisieren & $2 a / b \_9$ \\
\hline fterujizo & flattern & keine \\
\hline kapnízo1 & rauchen, Rauch ausgeben & $1 * a / b_{-} * 9$ \\
\hline kapnízo2 & räuchern & $2 a / b \_8 a / b \_9$ \\
\hline kapnízo3 & rauchen (etwas) & $8 a / b \_9$ \\
\hline ksinízol & sauer machen & $1 a / b \_9$ \\
\hline ksinízo2 & einen sauren Geschmack im Mund haben & $2 * a / b \_9$ \\
\hline mavrízo & schwarz machen & $1 a / b \_9$ \\
\hline midhenízol & vernichten & $2 a / b \_9$ \\
\hline midhenízo2 & auf Null bringen, die Note ' 0 ' vergeben & $8 a / b \_9$ \\
\hline oplízo & bewaffnen, ausrüsten & $4 a / b \_8 a / b \_9$ \\
\hline orízol & bestimmen, festsetzen, definieren & $8 a / b \_9$ \\
\hline orízo2 & über j-n/etwas herrschen, verfügen & $8 a / * b+9$ \\
\hline orjizo & ärgern, verärgern & $2 a / b \_9$ \\
\hline plevrízol & ankern, anlegen & $1 * a / b \_9$ \\
\hline plevrízo2 & längsseits kommen & $8 a / b_{-} * 9$ \\
\hline potízol & bewässern & $8 a / b \_9$ \\
\hline potízo2 & tränken (ein Tier/einen Menschen) & $4 a / b \_8 a / b \_9$ \\
\hline potízo3 & sickern & $1 a / b \_2 a / b \_9$ \\
\hline prasinízol & grün machen & $1 a / b \_9$ \\
\hline prasinizo2 & anfangen, mit grüner Flora bedeckt zu werden & $1 * a / b \_9$ \\
\hline progízol & ausbuhen, niederbrüllen & keine \\
\hline progízo2 & scheuchen & $1 a / b_{-} * 9$ \\
\hline sixtirízol & obszön beleidigen & keine \\
\hline sixtirízo2 & wütend werden & $2 * a / b \_9$ \\
\hline skorpízol & zerstreuen, verstreuen (Objekte) & $1 a / b \_2 a / b \_8 a / b \_9$ \\
\hline skorpízo2 & auseinandertreiben (Menschen, Tiere) & $3 a / b \_4 a / b \_8 a / b \_9$ \\
\hline skorpízo3 & abgeben, aussenden (Geruch, Duft) & $2 a / b \_9$ \\
\hline skorpízo4 & verschwenden, vergeuden & $8 a / b \_9$ \\
\hline stigmatizo & beflecken, brandmarken & $2 a / b \_8 a / b \_9$ \\
\hline stolízol & zieren, schmücken & $8 a / b \_9$ \\
\hline stolizo2 & kleiden & $4 a / b \_8 a / ? b \_9$ \\
\hline sximatízol & formen, bilden & $2 a / b \_9$ \\
\hline sximatízo2 & zeichnen & $2 a / b \_8 a / b \_9$ \\
\hline
\end{tabular}




\begin{tabular}{|l|l|l|}
\hline termatízol & beend(ig)en & $2 a / b \_8 a / b \_9$ \\
\hline termatízo2 & durchs Ziel gehen & keine \\
\hline thrimatízo & zerbrechen, fragmentieren & $2 a / b \_8 a / ? b \_9$ \\
\hline tiganízo & in der Pfanne braten & $8 a / b \_9$ \\
\hline tonízo1 & betonen, akzentuieren, hervorheben & $8 a / b \_9$ \\
\hline tonízo2 & sich herausheben, sich abheben & $2 a / b \_9$ \\
\hline travlízo & stottern & $k e i n e$ \\
\hline vrízo & beschimpfen & $6 a / b \_8 a / b \_9$ \\
\hline vurtsízo & bürsten & $8 a / b \_9$ \\
\hline xeretízo1 & "Hallo" sagen, begrüßen & $6 a / b \_* 9$ \\
\hline xeretízo2 & mit Zustimmung/Freude empfangen, begrüßen & $8 a / b \_* 9$ \\
\hline xtenízo & kämmen & $4 a / b \_8 a / b \_9$ \\
\hline
\end{tabular}




\section{Appendix B: Neugriechische Verbendungen ${ }^{a}$}

\begin{tabular}{|c|c|c|c|c|c|c|}
\hline \multirow[b]{2}{*}{ Active voice: } & \multicolumn{2}{|c|}{$1^{\text {st }}$ conjugation } & \multicolumn{2}{|c|}{$\begin{array}{l}2^{\text {nd }} \text { conjugation: } \\
\text { type A }\end{array}$} & \multicolumn{2}{|c|}{$\begin{array}{l}2^{\text {nd }} \text { conjugation: } \\
\text { type } B\end{array}$} \\
\hline & $S g$. & $P l$. & $S g$. & Pl. & $\mathrm{Sg}$ & $P l$. \\
\hline \multirow[t]{3}{*}{ Present } & '-o & '-ume & -ó & -áme & -ó & -úme \\
\hline & -is & '-ete & -ás & -áte & -ís & -íte \\
\hline & $-i$ & -un & -ái & -ún & -1 & -ún \\
\hline \multirow[t]{3}{*}{ Imperfect } & $--\mathrm{a}$ & 'ame & -úsa & -úsame & -úsa & -úsame \\
\hline & '--es & -ate & -úses & -úsate & -úses & -úsate \\
\hline & $'--\mathrm{e}$ & $'-$ an & -úse & -úsan & -úse & -úsan \\
\hline \multirow[t]{3}{*}{ Dependent } & '-o & '-ume & -0 & '-ume & -0 & '-ume \\
\hline & '-is & '-ete & '-is & '-ete & '-is & '-ete \\
\hline & $'-i$ & '-un & ${ }^{\prime}-\mathrm{i}$ & '-un & ${ }^{\prime}-\mathrm{i}$ & '-un \\
\hline \multirow[t]{3}{*}{ Simple past } & $--\mathrm{a}$ & 'ame & '--a & 'ame & $2-a$ & '-ame \\
\hline & '--es & -ate & '--es & -ate & '--es & '-ate \\
\hline & '--e & '--an & '--e & '--an & '--e & '--an \\
\hline $\begin{array}{l}\text { Imperfective } \\
\text { imperative }\end{array}$ & ${ }^{\prime}--e / l^{\prime}-e$ & '-ete & $'-\mathrm{a}$ & -áte & $'-i$ & -íte \\
\hline $\begin{array}{l}\text { Perfective } \\
\text { imperative }\end{array}$ & $\prime--e / l^{\prime}-e$ & '-(e)te & '--e & -te & '-e & -te \\
\hline Gerund & & das & & das & & das \\
\hline
\end{tabular}

\begin{tabular}{|c|c|c|c|c|c|c|}
\hline Passive voice: & $S g$ & $P l$. & $\mathrm{Sg}$ & Pl. & $S g$ & $P l$. \\
\hline \multirow[t]{3}{*}{ Present } & '-ome & -ómaste & -iéme & -iómaste & -úme & -úmaste \\
\hline & '-ese & '-este & -iése & -iéste & -íse & -íste \\
\hline & '-ete & '-ode & -iéte & -iúde & -íte & -úde \\
\hline \multirow[t]{3}{*}{ Imperfect } & -ómun & -ómastan & -iómun & -iómastan & -úmun & -úmastan \\
\hline & -ósun & -ósastan & -iósun & -iósastan & -úsun & -úsastan \\
\hline & -ótan & -odan & -iótan & -iúdan & -údan & -údan \\
\hline \multirow[t]{3}{*}{ Dependent } & -ó & -úme & -ó & -úme & -ó & -úme \\
\hline & -ís & -íte & -ís & -íte & -ís & -íte \\
\hline & -1 & -ún & $-1 ́$ & -ún & -í & -ún \\
\hline \multirow[t]{3}{*}{ Simple past } & -ika & -íkame & '-ika & -íkame & '-ika & -íkame \\
\hline & '-ikes & -íkate & '-ikes & -íkate & '-ikes & -íkate \\
\hline & -ike & '-ikan & 'ike & '-ikan & -ike & '-ikan \\
\hline $\begin{array}{l}\text { Imperfective } \\
\text { imperative }\end{array}$ & \multicolumn{2}{|c|}{ (lacking) } & \multicolumn{2}{|c|}{ (lacking) } & \multicolumn{2}{|c|}{ (lacking) } \\
\hline $\begin{array}{l}\text { Perfective } \\
\text { imperative }\end{array}$ & $-\mathrm{u}$ & -íte & $-\mathrm{u}$ & -íte & $-\mathrm{u}$ & -íte \\
\hline
\end{tabular}

${ }^{a}$ Aus: Holton, David/Mackridge, Peter/Philippaki-Warburton, Irene (1997): Greek: a comprehensive grammar of the modern language, London, S. 116. Diese angepaßten Tabellen werden hier mit Genehmigung angeführt. 